\title{
Optics in engineering education: stimulating the interest of first-year students
}

Jesús Blanco-García, Benito Vazquez-Dorrío

Jesús Blanco-García, Benito Vazquez-Dorrío, "Optics in engineering education: stimulating the interest of first-year students," Proc. SPIE 9289, 12th Education and Training in Optics and Photonics Conference, 92890Q (17 July 2014); doi: 10.1117/12.2070765

SPIE Event: 12th Education and Training in Optics and Photonics Conference, 2013, Porto, Portugal 


\title{
Optics in engineering education: stimulating the interest of first-year students
}

\author{
Jesús Blanco-García, Benito V. Dorrío \\ University of Vigo, Applied Physics Dept., EEI, Torrecedeira 86, 36208 Vigo, Spain
}

\begin{abstract}
The work here presented deals with stimulating the interest for optics in first-year students of an Engineering School, which are not specifically following Optical Engineering studies. Optic-based technologies are nowadays wide spread, and growing, in almost all the engineering fields (from non destructive testing or alignments to power laser applications, fiber optic communications, memory devices, etc.). In general, the first year curriculum doesn't allow a detailed review of the main light properties, least its technical applications. We present in this paper our experience in showing some basic optic concepts and related technologies to the students of our school. Based on the fact that they have a very basic training in this branch of physics, we have designed a series of experimental demonstrations with the dual purpose of making them understand the basic principles of these technologies, and to know the potential of applications to engineering they offer. We assembled these experiments in the laboratory and invited students to pass to get to know them, giving them an explanation in which we focused on the possible range of application of each technique. The response was very good, not only by the number of students who attended the invitation but also by the interest demonstrated by their questions and opinions.
\end{abstract}

Keywords: Engineering education, optics education.

\section{INTRODUCTION}

The scientific knowledge of the light nature and its handling results nowadays in a multitude of techniques to solve many problems in all engineering fields. From metrology, alignment or non destructive testing to power applications for cutting or welding there is a wide technological field that can't be absent from the engineer education curricula. Not only the optical engineer student needs an adequate formation in optics related issues but also those who want acquire competence in the wide field of the modern technology have to be familiarized with the Optic principles and applications [1].

In our case, we teach Physics for different engineer specialties, none of them Optical Engineering. Physics is part of the core curriculum and is taught in the first year. Optics is included in the Physics program, but as it has to be developed in only four hours per week (including laboratory training), little time can be really devoted to an in-depth study of even the basic optical concepts. This, and the fact that the authors' particular research field is an optical application (Metrology), is the reason that led us to design an extra-program activity to encourage students to learn about optics and its technical applications. To attain this goal we designed a series of laboratory experiments that cover different aspects of the optical science (geometric optics, interference and diffraction, polarity, laser, etc.), single enough to be understood by first year's students with very basic optical concepts. They are invited to visit our laboratory, to attend our explanations and eventually to participate in the performance of these experiences. We try to show both, the basic principles in which each experiment relies and the potential application that it allows. In this sort of workshop we include a visit to our research laboratory (Fig. 1.a, Fig. 1.b) in order to show non-educational experimental arrangements to the students (mainly interferometers for different applications [2-3]) and provide information about the MSc on Photonics and Laser Technologies where the authors are part of the academic team [4]. This allows students to understand the importance of the research activity also in the engineering field. Summarizing, in this work we have tried to place the student in front of a series of experimental facts that should constitute "an amazement” for him, and hence, a starting point in his learning process.

In general, the students answer was very good; they show a clear interest in the proposal: a large number of them attended this activity, collaborating in the development of the experiments, asking questions, etc.

12th Education and Training in Optics and Photonics Conference, edited by

Manuel F. P. C. Martins Costa, Mourad Zghal, Proc. of SPIE Vol. 9289, 92890Q

(C) 2014 SPIE, OSA, IEEE, ICO · doi: 10.1117/12.2070765

Proc. of SPIE Vol. 9289 92890Q-1 


\section{DESCRIPTION OF THE EXPERIENCES}

As it was explained before, in the design of these experiences we took into account both the necessity to show in a clear way the basic principles and phenomena, and also to suggest their potential Engineer applications. Indeed, as we could see, it is the possibility of use of the optical technologies in their future professional fields that really encouraged the students interested; but our goal was not only they were able to appreciate the possibilities of practical use of the optics in engineering, we have also tried to make them understand, at least in a basic way, the optical fundamental concepts. We believe that the University engineering education should highlight its scientific basis and be not limited to training the students as future users of a particular technology. We want students to gain the idea that scientific knowledge, in principle very generic, are always at the root of any technological field. As one further stimulating element, we have structured the experiences surrounding the common theme of the laser: its structure as a light source, the properties of its radiation, applications, etc. The basic differences between the laser light, coherent, and that emitted by other light sources has served us also as a thread to our explanation and to the different discussions so originated. According to this, it has to be covered a wide panoramic view ranging from the elucidation of basic scientific concepts to show some technical applications. This dual approach entails two requirements:

- Simplicity in the experimental set-up. This should highlight the phenomenon or principle which is being illustrating, in a way avoiding to deflect the attention of the student to the technical details of the instrumentation.

- Relationship as closer as possible with some technical application of the illustrated concept. It is desirable even to highlight, if it were the case, some relationship between the experience and the everyday experience of the pupil (interference fringes in a film of oil on water or soap films [5] (Fig. 1.c), rainbows (Fig 1.d) [6], moiré effects caused by the interaction of layers of periodic clothes (Fig. 1.e) [7], etc.).

We should also highlight that, given the low level of the pupil's knowledge in optics, to whom these experiences are addressed, as noted in the previous section, and the short last of the visit to the laboratory (in an hour at most), we have not tried to deepen in each experience as much as it would be possible. In addition, and for the same reasons, nor do we intend, in this trial or laboratory prototype, that the student perform himself experiences and measures, thus having the experimental arrangements a demonstrative character.

\subsection{Reflection and Refraction}

As a starting point, it seems natural to take the refraction (Fig. 1.f) and reflection (Fig. 1.g) phenomena. This allows to show clearly the basic laws of the light propagation and to introduce also the geometrical optics principles. The experience we implemented consisted in a transparent and elongated methacrylate bucket with a solution of sugar in water, and a laser pointer (Fig. 1.h). Pouring water slowly on a layer of two or three centimeters of sugar in the bucket bottom, and waiting for a couple of days that this dissolves, it is obtained a dissolution with a gradient of concentration in the bottom of the bowl and, therefore, a refractive index gradient medium. The laser beam path becomes directly observable by adding a few drops of milk to the solution, which give us the opportunity to explain the scattering phenomenon (Figure 2). In a first approach, we show the change of the beam direction when passing from air to water, and make the pupils note also the fact that part of the light passes from one medium to another and part is reflected [8]. Subsequently the laser beam can be made pass from the water to the air and show how in this case the refracted angle is larger than the incidence one, in such a way that increasing this last one the refraction reaches the ninety degrees and the total reflection is attained. The most interesting thing in this arrangement is to show how the light is bent in passing through a gradient index medium. In this point, it is interesting to explain the formation of some mirages that can be observed easily (for example in a road heated by the sun in summer or on the sea [9]), in the same way some application of gradient index can be mentioned (optical fibers and integrated optics for example).

\subsection{Polarization and Photoelasticity}

The next topic that we showed to our students is the polarization (Fig. 1.i). To introduce them in it, we explain the difference between a longitudinal and a transverse wave first using the analogy of waves in a long spring. We made the students to see how a transverse wave can have a defined plane of vibration and how a polarizer can filter all the components of the amplitude vector except one (we have restricted to the polarization by absorption). Next, by using to polarizer plates the students could see the light polarization phenomenon. The photoelasticity was observed introducing a 
transparent plastic object such as a CD case or a protractor (Fig. 1.j), between the two polarizers, so residual strengths become observable. In the same way, the students themselves can apply a mechanical load to the object and observe the stress distribution on it. In addition, they could probe that the laser light is polarized with the HeNe we use for this experiences. As practical application, apart from the polarized sunglasses and photography polarizing filters, it was mentioned the study of stress distributions in structures by analyzing the photoelastic behavior of transparent methacrylate models [10].
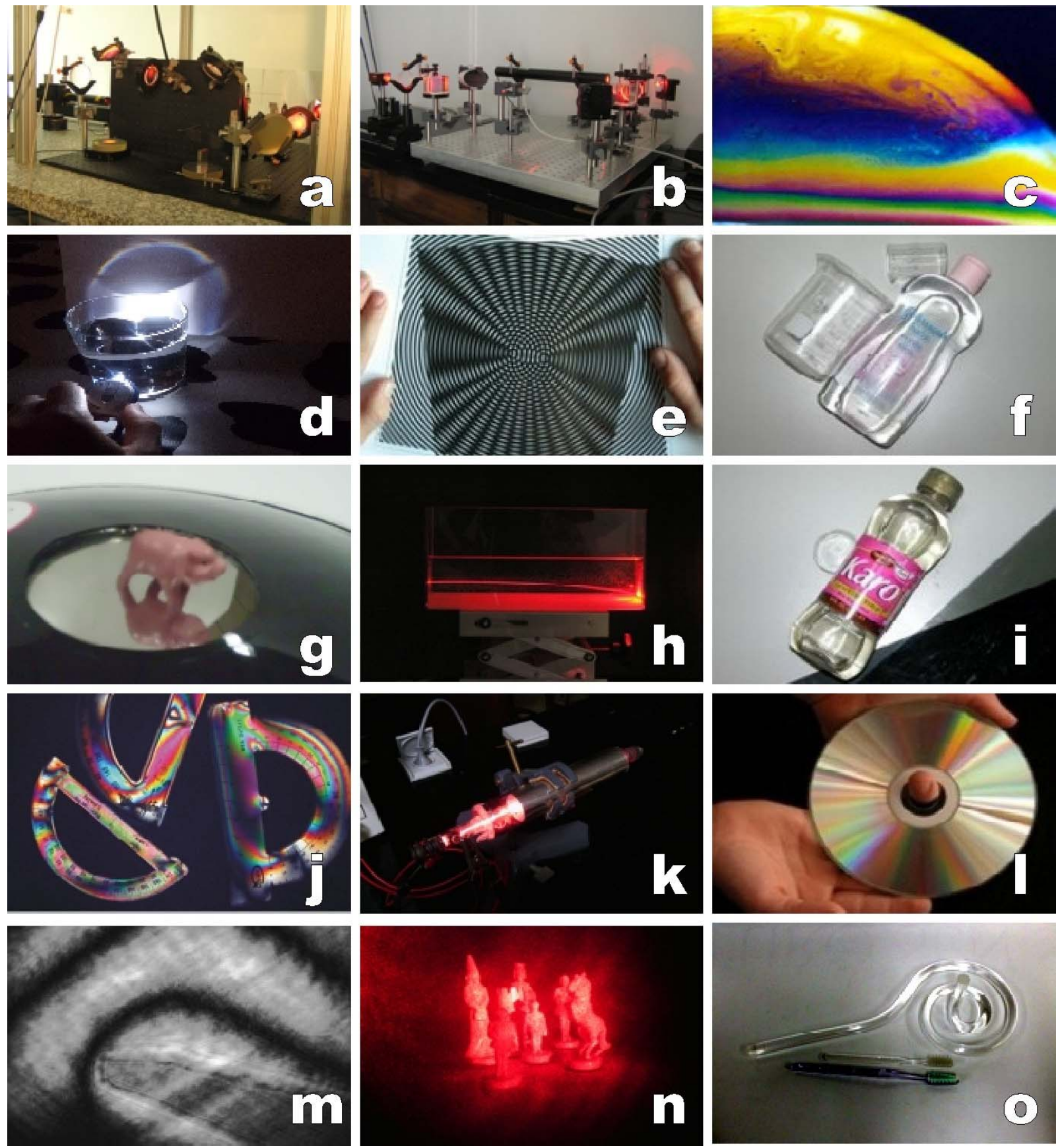

Figure 1. A selection of the activities carried out during the experience. 


\subsection{HeNe Laser Tube}

The principle and operation of a laser was explained using a "nude" HeNe laser tube (Fig. 1.k), where the electric discharge can be observed and also the resonance cavity and its emission. It is powered with a high voltage source. With the aid of several diagrams, we explain the population inversion and the stimulated emission concepts and how they determine the basic properties of the laser light. This experience also allows describing the different classifications of lasers attending the active medium, the pumping system, kind of emission, etc. Many laser applications are really wide known and this gives the opportunity to make participate the students talking about the practical cases each one can describe [11].

\subsection{Diffraction}

One of the most important properties of light as a wave is the diffraction phenomenon. Using a laser pointer and a transmission diffraction grating, we see how the incident beam is split and the diffraction pattern that is formed. We underline, by using different gratings, that the value of the diffraction angle for each order depends on the wavelength of the used light and on the number of lines per mm of the grating (i.e., the width of the slits). Next, we show the diffraction pattern produced by a hair, and suggest the idea that it is possible to measure the thickness of it by measuring the separation between the bright spots of the diffraction pattern and make pupils note that simply to move away the observation screen from the grating improves the accuracy of measurement, since it decreases the uncertainty in the determination of the diffraction angle. As an industrial application we mentioned the in-line measurement employed as quality control in the manufacture of different kind of wires.

As an example of reflection grating we showed simply a CD (Fig. 1.l). When a laser beam impinges on its surface a diffraction pattern is clearly observable, due to the effect of pits and lands that codify the binary information. As the grooves describe a spiral, the diffraction pattern is seen also curved. Next, the CD is illuminated with a white light focus and pupils can observe the different behavior respect to the monochromatic light, since in this case the pattern consists in color series ranging all the visible spectrum [12].

\subsection{Interference and speckle}

To explain the interference phenomenon, the students were drive to the Optical Metrology Laboratory, where we have currently two interferometer arrangements. One of them is a Twyman-Green with two wavelengths in a vertical configuration, for gauge-block measurements, and the other is a Mach-Zehnder for fluid flow analysis. With the aid of several draws, the basic concept was exposed, the different parts of the interferometers were described and the resulting fringe pattern was shown. We begin by explaining how takes place the division and recombination of the beam in both interferometers and clarify that the superposition of two beams of coherent light can give rise to areas without light (dark stripes) depending on the difference of the path traveled by each beam. One of the mirrors is mounted on a translation stage drove by micrometric screws, so that the students themselves can see how very a small displacement of the mirror are magnified in the displacement of the interference pattern, and acquire so an idea of the sensibility of the method. This sensibility can also be demonstrated by asking them to bring a hand close to an arm of the interferometer after being it rub, and observe the distortion of the fringes due to the small air convention produced (Fig. 1.m). The effect of a fluid flow in the Mach-Zehnder, with and without carrier fringes was also shown. Several industrial applications of the interferometers, for example in quality control, were discussed [13].

The speckle phenomenon as a consequence of the coherent light emitted by a laser was also shown here. The speckle effect is observed over a rough surface object (or a transmission diffuser) when it is illuminated by a beam of coherent light. It's a pattern of interference due to the overlap in every point in the space of a large number of wave fronts with random phase differences, scattered by a rough surface. Focusing a laser beam on a rough surface or passing through a diffuser this effect is easily observable. The pupils should realize that this phenomenon is three-dimensional, filling a volume around the object. This can be seen easily by observing the movement of the speckle pattern respect the object surface when, without glasses, they move the head: those who are short-sighted will see it moving in the opposite direction than their own movement and the hyperopic ones will observe the speckle pattern moves in their own direction (those who have healthy sight will see this effect if there wear the glasses of a mate). Subsequently, we discussed that this effect would make portraits with coherent light unfeasible, but some applications like non destructive testing can take advantage of this non-everyday phenomenon [14]. 


\subsection{Holography}

We include holography (Fig. 1.n) in this series of experiences because it is an issue of undoubted importance in modern optics and by being fairly reported, although, for reasons of conceptual complexity, we could not deal with their technical applications (with the exception of the holographic interferometry) neither to explain how we would like its scientific foundations. As expected, it was one of the experiences that most caught his attention.

We showed them a transmission hologram reconstructed with HeNe laser. The reproduced scene consisted of a series of chess pieces placed in different positions, the image was virtual and at certain distance behind the plate. First, did them note that the relative position of the parts change if they move, exactly the same as with real objects. They realized that this does not happen with a photograph, and that a hologram is closer to a framework through which we look at a scene than to a picture.

To the groups of students that showed more curiosity we tried to explain, with the help of some schemes, that what there is in the holographic plate is not an image of the object but a pattern of interference. This pattern is originated by the superposition of the light coming from the object (object wave front) and the reference beam. Once registered this pattern in the photosensitive medium, it constitutes a diffraction grating. When laser light passes through it (generally the reference beam), it is diffracted: one of the diffraction orders reconstructs the object wavefront. The possibility to emulate this optical principle by a calculation process in a computer (Digital Holography) is also mentioned.

Finally, it was discussed that using this technique it is possible to superimpose two wavefronts coming from the same object in two different deformation states (for example, loaded and unloaded). In this way an interference pattern is produced in which the fringe form and spacing is function of the deformation. This is the Holographic Interferometry technique which allows applying the intereference principle to diffusing surfaces. A series of industrial application of it was discussed: automotive industry, aircraft, etc [15].
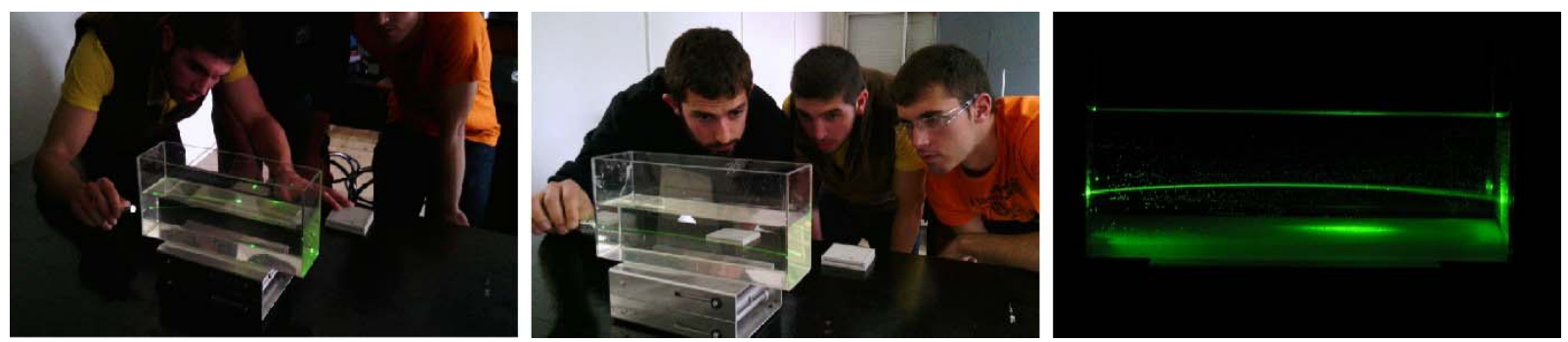

Figure 2. Participants during the activity.

\subsection{Optical Fiber}

The experience of total reflection helps us to introduce the concept of optical fiber. With the help of a scheme we explain how, for a certain cone of incidence of the light in an optical fiber, rays can be confined inside it, and can thus travel all along the fiber (Fig. 1.0).

As an example, we have used a plastic multimode fiber, launching the laser beam inside it using a converging lens. As in fact all fiber appears illuminated, it was necessary to clarify that this was due to impurities in the material losses, (naturally, the light went out of fiber by the other end with significant intensity). Based on this, we explained briefly the applications of optical fiber in communications, sensors (mechanical stress, temperature, density, pH, etc.) and endoscopes [16].

\section{RESULTS AND CONCLUSIONS}

The result of this work of motivation of the students towards the applications of optics in engineering can be frankly considered as satisfactory. To analyze their interest towards this proposal, we carried out a survey whose results appeared to us to be very encouraging (was performed both among students who attended the laboratory as well as among those who did not): 
- $100 \%$ of the students considered interesting to have at least some basic knowledge of optics to apply in their future professional field;

- $86 \%$ of the students considered interesting the possibility of studying an optional subject related to the subject;

- $\quad 93 \%$ of the students saw at least interesting the possibility of doing his thesis on optics applications to engineering.

- $100 \%$ of those who did not attend claim not to have done by not have learned of the call to visit the laboratory or have not been able for reasons of time, never due to lack of interest.

We believe that these results reflect that this experience was able to create a climate of concern and interest in light, laser and photonics applications in engineering.

\section{REFERENCES}

[1] Cloud, G., [Optical Methods of Engineering Analysis], Cambridge University Press, New York (1998).

[2] Diz-Bugarín, J., Dorrío, B.V., Blanco, J., Yebra, F. J., Outomuro, I., Otero, M., Rodríguez, J. Miranda, M., Valencia, J. L., "Study of the refractometric stability of an interferometer equipment for gauge block calibration", Proc. SPIE 8493, 8493161-8493168 (2012).

[3] Rodríguez, R., Dorrío, B.V., Blanco, J., "Evaluation of interferometric patterns of supersonic fluid flows by the differential Fourier transform method”, Proc. SPIE 8493, 8493181-84931810 (2012).

[4] http://master.laserphotonics.org/eng/descripcion.html

[5] Rämme, G., “Colors on Soap Films. An Interference Phenomenon”, Phys. Teach. 28, 479-480 (1990).

[6] Russell, R.D., “A rainbow for the classroom”, Phys. Teach. 27, 262-263 (1989).

[7] Bernero, B., “The Moire Effect in Teaching Physics”, Phys. Teach. 27, 602-608 (1989).

[8] Richey, L., Stewart, B. and Peatross, J., “Creating and analyzing a mirage”, Phys. Teach., 44, 460-464 (2006).

[9] Blanco-García, J., Dorrío, B.V., Ribas-Pérez, F.A., "Photographing mirages above the sea surface”, Proc. Hsci2011, 78-85 (2011).

[10] Ellenstein, M., “The Broken-Protractor Prediction Trick”, Phys. Teach. 40, 52 (2002).

[11] Ready, J.F., "Properties and Applications of Lasers", Phys. Teach. 6, 344-351 (1968).

[12] Greenslade, T.B., “Diffraction by a cat's whisker”, Phys. Teach. 38, 422 (2000).

[13] Vollmer, M., Möllmann, K. P., “Michelson Interferometer for Your Kitchen Table”, Phys. Teach., 46, 114-117 (2008).

[14] Carlsten, J.L., “Laser Speckle”, Phys. Teach. 25, 175-176, (1987).

[15] Spetzler, H., “Interesting Examples of Holographic Interferometry”, Phys. Teach. 24, 80- 85 (1986).

[16] Edgar, D., “Simulating optical fibers”, Phys. Teach. 26, 504-506 (1988). 